\title{
Modeling and Simulation of Low Power Ferroelectric Non-Volatile Memory Tunnel Field Effect Transistors Using Silicon-doped Hafnium Oxide as Gate Dielectric ${ }^{\text {th }}$
}

\author{
A. Saeidi ${ }^{1, *}$, A. Biswas, Adrian M. Ionescu \\ Laboratory of Micro and Nano-electronic Devices, Ecole Polytechnique Fdrale de Lausanne, \\ Lausanne, Switzerland
}

\begin{abstract}
The implementation and operation of the nonvolatile ferroelectric memory (NVM) tunnel field effect transistors with silicon-doped $\mathrm{HfO}_{2}$ is proposed and theoretically examined for the first time, showing that ferroelectric nonvolatile tunnel field effect transistor (Fe-TFET) can operate as ultra-low power nonvolatile memory even in aggressively scaled dimensions. A Fe-TFET analytical model is derived by combining the pseudo 2-D Poisson equation and Maxwell's equation. The model describes the Fe-TFET behavior when a time-dependent voltage is applied to the device with hysteretic output characteristic due to the ferroelectric's dipole switching. The theoretical results provide unique insights into how device geometry and ferroelectric properties affect the Fe-TFET transfer characteristic. The recently explored ferroelectric, silicon-doped $\mathrm{HfO}_{2}$ is employed as the gate ferroelectric. With the ability to engineer ferroelectricity in $\mathrm{HfO}_{2}$ thin films, a high-K dielectric well established in memory devices, the silicondoped $\mathrm{HfO}_{2}$ opens a new route for improved manufacturability and scalability of future 1-T ferroelectric memories. In the current research, a $\mathrm{Si}: \mathrm{HfO}_{2}$ based Fe-TFET with large memory window and low power dissipation is designed and simulated. Utilizing our presented model, the device characteristics of a FeTFET that takes full benefits from $\mathrm{Si}: \mathrm{HfO}_{2}$ is compared with the same devices
\end{abstract}

\footnotetext{
This document is a collaborative effort.

* Corresponding author

Email address: ali.saeidi@epfl.ch (A. Saeidi)
} 
using well-known perovskite ferroelectrics. Finally, the Fe-TFET is compared with a conventional ferroelectric memory transistor highlighting the advantages of using tunneling memory devices.

Keywords: $\mathrm{HfO}_{2}$, analytical model, surface potential, ferroelectric, nonvolatile memory, Fe-TFET

\section{Introduction}

Ferroelectric materials can be utilized as electrically switchable nonvolatile data storage elements as their polarization can change by applying an external electric field. Recently, novel devices called ferroelectric TFETs [1, and ferro5 electric FETs have been proposed as promising candidates for future nonvolatile memory applications [2, 3]. In ferroelectric field effect devices, two stable states of the ferroelectric's polarization are used for data storage [4]. Nonvolatile data storage, fast writing, and nondestructive read-out operation have been reported for ferroelectric field effect transistors [5. However, the industrial implementation of ferroelectric devices especially in nanoscale still missing due to the integration and scaling obstacles of conventionally used perovskite type ferroelectrics such as Lead Zirconate Titanate (PZT). The recently discovered ferroelectricity in $\mathrm{HfO}_{2}$ thin films [6], enabled CMOS-compatible manufacturing of highly scaled ferroelectric devices down to $28 \mathrm{~nm}$ ground rule [6, 7].

By scaling devices down to the nanoscale, power density becomes a challenging issue as the power density per area of the chip increases. The tunneling field effect transistors have been investigated intensely in recent years 8 due to their considerable potentials for ultra-low power applications [9, 10]. Using the advantages of both ferroelectric thin films for data storage and TFETs as energy efficient devices, it is possible to design a new class of nonvolatile memories, with a relatively large memory window, fast writing, nondestructive read-out operation, and low power consumption. In this work, we propose a comprehensive, quantitative model for investigation of Fe-TFETs. The successful design of a Fe-TFET for nonvolatile data storage requires a thorough understanding of 


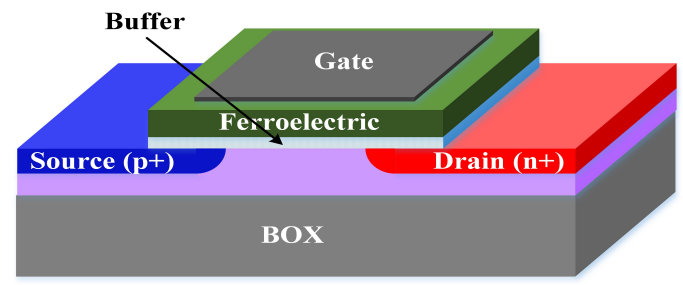

Figure 1: The device schematic of an SOI Fe-TFET where the gate stack of a conventional single gate TFET is replaced with a series combinations of a ferroelectric and a linear dielectric.

the device operation principals. It is necessary to develop an analytical model for Fe-TFETs design optimization. Hence, an analytical model for Fe-TFETs is proposed and verified with Sentaurus TCAD [11] simulation tool. The model is developed by solving the 2-D Poisson equation [12 for a single gate TFET and considering the effect of the ferroelectric polarization on the surface poten-

so tial. The polarization effect is calculated by solving Maxwell's first equation in the gate stack. A numerical method is employed to calculate the polarization hysteresis for both saturated and nonsaturated hysteresis loops [13. Based on our proposed model, memory window of the ferroelectric memory TFETs using different ferroelectric materials is investigated. It should be noted that short channel effects are neglected in the proposed model, and a $200 \mathrm{~nm}$ gate length device is utilized for model verification. Finally, a $28 \mathrm{~nm}$ gate length Fe-TFET benefits from $\mathrm{Si}: \mathrm{HfO}_{2}$ as the gate ferroelectric is designed and simulated using Sentaurus TCAD commercial simulator. Results confirm that the proposed device can be employed as the future of nonvolatile memories. Moreover, we 40 present that the ferroelectrics negative capacitance improves the device's output current by boosting the gate potential.

\section{Device Modeling}

\subsection{Approach and Definitions}

In this paper, an n-type SOI ferroelectric TFET is studied. The device is 45 schematically depicted in Fig. 1. The length of the channel is $200 \mathrm{~nm}$ to suppress 
short channel effects, the doping concentration of the lightly doped channel is $5 \times 10^{14} \mathrm{~cm}^{-3}$, the highly doped $p+$ source concentration is $1 \times 10^{20} \mathrm{~cm}^{-3}$ and the $n+$ doped drain is $1 \times 10^{18} \mathrm{~cm}^{-3}$ to eliminate the ambipolar behavior [13]. The silicon-doped $\mathrm{HfO}_{2}$ is utilized as ferroelectric layer due to its unique property of exhibiting the ferroelectricity even in a $5 \mathrm{~nm}$ thick, thin film [14, 15]. For the model verification, we have used $10 \mathrm{~nm}$ of $\mathrm{Si}: \mathrm{HfO}_{2}$ with $9 \mu \mathrm{C} / \mathrm{cm}^{2}$ remanent polarization and $1.1 \mathrm{MV} / \mathrm{cm}$ coercive field [6]. We will discuss how the relatively high coercive field and remanent polarization guarantee a large memory window (and retention time) in the Ferroelectric Memory TFET.

To develop the ferroelectric TFET model, we have combined the switching analytical modeling of ferroelectric capacitors [16] with the Band-to-Band Tunneling modeling of TFETs. Wu et al. [12] proposed the analytical modeling of TFETs based on the gate and drain dual modulation effects by solving Poisson equation in the device channel. Miller et al. [17, 13, 18, developed the compact modeling of ferroelectric capacitors that accurately describes the dipole polarization switching by employing Maxwell's first equation. We employed Maxwell and Poisson equations consistently to precisely calculate the surface potential profile along the channel. Finally, the device current is calculated by determining the potential profile around the tunneling junctions. The 65 computer aided design (TCAD) tool Sentaurus is used to verify the proposed model. The non-local band-to-band tunneling (BTBT) model is enabled to account tunneling mechanism [19]. In order to calculate the tunneling probability using the electron-hole wave vector throughout the tunneling path, the nonlocal model uses Wetzel-Kramer-Brillouin (WKB) approximation. Also, Fermi statistics and Shockley-Reed-Hall (SRH) recombinations are adopted while the gate tunneling is ignored. In order to incorporate the ferroelectric properties of the gate stack, we have enabled Ferro model in our simulations. Moreover, the standard library of Sentaurus TCAD is used for linear dielectrics (where the $\mathrm{SiO}_{2}$ and $\mathrm{Si}_{3} \mathrm{~N}_{4}$ relative permittivity is 3.9 and 7.5 respectively). These models 75 are considered in all TCAD simulations unless otherwise mentioned. It should be noted that the ferroelectric layer is considered ideal from the point of view of 
hysteresis loss related issues like fatigue (loss of switched charge owing to repetitive destructive reads), retention (decreasing the stored charge to a level where the positive and negative state of the polarization cannot be reliably sensed), and direct current breakdown (as a result of applying constant writing voltage) have been neglected [20].

\subsection{Model}

In this subsection, we describe the relation between the ferroelectric polarization and the silicon surface potential in a metal-ferroelectric-oxide-semiconductor structure 21. The electrostatic equations are derived starting with Maxwell's equation,

$$
\begin{gathered}
\nabla \cdot D=\rho, \\
D=\epsilon_{0} \epsilon E+P_{d},
\end{gathered}
$$

where $D$ is the displacement, $\rho$ is the free charge density, $E$ is the electrical field, $\epsilon_{0}$ is the vacuum permittivity, $\epsilon$ is the linear dielectric constant, and $P_{d}$ is the contribution of the switching dipoles. Solving (1) and (2) in conjunction with the definition $E=-\nabla \phi$ ( $\phi$ is the electrostatic potential) leads us to:

$$
V_{G B}=\phi_{s}-\frac{\sigma_{s}}{C_{\text {stack }}}-P_{d}\left(E_{f}\right) \frac{d_{f}}{\epsilon_{0} \epsilon_{f}} .
$$

In (3), $C_{\text {stack }}$ describes the gate total capacitance, $\sigma_{s}$ is the silicon charge, $d_{f}$ and $\epsilon_{f}$ are ferroelectric thickness and relative permittivity and $\phi_{s}$ is the silicon surface potential. The ferroelectric polarization changes the surface potential by a value of $P_{d}\left(d_{f} / \epsilon_{0} \epsilon_{f}\right)$. The ferroelectric polarization can also be calculated using Landau-Khalatnikov (LK) theory [22, 23, 24]. Using Landau theory, (3) can be rewritten as follow:

$$
V_{G B}-\phi_{s}+\frac{\sigma_{s}}{C_{B u f f e r}}=\left(2 \alpha t_{f}\right) P_{d}\left(E_{f}\right)+\left(4 \beta t_{f}\right) P_{d}^{3}\left(E_{f}\right)+\left(6 \gamma t_{f}\right) P_{d}^{5}\left(E_{f}\right),
$$


where $\alpha, \beta$, and $\gamma$ are the ferroelectric Landau parameters, $t_{f}$ is the ferroelectric thickness, and $C_{B u f f e r}$ is the capacitance of the buffer layer. The Fe-TFET modeling can be obtained using either (3) or (4). However, (3) is employed in this study to derive a simple analytical model for the device operation.

We explain the Fe-TFET behavior by considering the effect of the polarization on the surface potential for a known polarization. Combining Maxwell's first equation with Wu's analytical solution for TFET surface potential [12], the surface potential in middle of the channel for the whole range of the operation can be given as

$$
\begin{aligned}
& \phi_{c h}= F+\frac{k T}{q} \ln \left\{\frac { q } { k T } \left[\frac{k T}{q}+\frac{\sqrt{F}}{\sqrt{F}+\gamma}\left(V_{G S}-V_{f b}+P_{d} \frac{d_{f}}{\epsilon_{0} \epsilon_{f}}-F\right)+\right.\right. \\
&\left.\left.\frac{1}{2}\left[\frac{F}{(\sqrt{F}+\gamma)^{2}}-\frac{\gamma[F-2]}{2(\sqrt{F}+\gamma)^{3}}\right]\left(V_{G S}-V_{f b}+P_{d} \frac{d_{f}}{\epsilon_{0} \epsilon_{f}}-F\right)^{2}\right]\right\} \\
& F= \frac{1}{2}\left\{V_{D S}+\Phi+\phi_{c h, d e p}-\sqrt{\left(\phi_{c h, d e p}-V_{D S}-\Phi\right)^{2}+\delta^{2}}\right\}, \\
& \phi_{c h, d e p}=\left\{\sqrt{V_{G S}-V_{f b}+P_{d} \frac{d_{f}}{\epsilon_{0} \epsilon_{f}}+\frac{\gamma^{2}}{4}}-\frac{\gamma}{2}\right\}^{2},
\end{aligned}
$$

where $V_{f b}$ is the flat band voltage, $T$ is the temperature, $k$ is the Boltzmann's constant, $q$ is the electrical charge of a single electron, $\gamma$ is the body factor defines as $\sqrt{2 \epsilon_{s i} q N_{c h}} / C_{\text {stack }}$ ( $N_{c h}$ is the channel doping), $\delta$ is a small smoothing factor and $\phi_{c h, d e p}$ is the surface potential in the gate control regime [12]. $\Phi$ is the required surface potential for sufficient inversion charge to screen the gate modulation and can be expressed as $(k T / q) \ln \left(N_{c h} N_{i n v} / n_{i}^{2}\right)$, and $N_{i n v}$ is the required inversion charge density to screen the gate voltage.

An accurate expression of the surface potential profile around the tunnel junction is needed to calculate the tunneling current. We use the pseudo 2-D Poisson equation to obtain the potential profile along the channel [25]. The parabolic approximation of the potential in a direction normal to the surface is adopted so that the Poisson equation can be reduced to the well-known form 


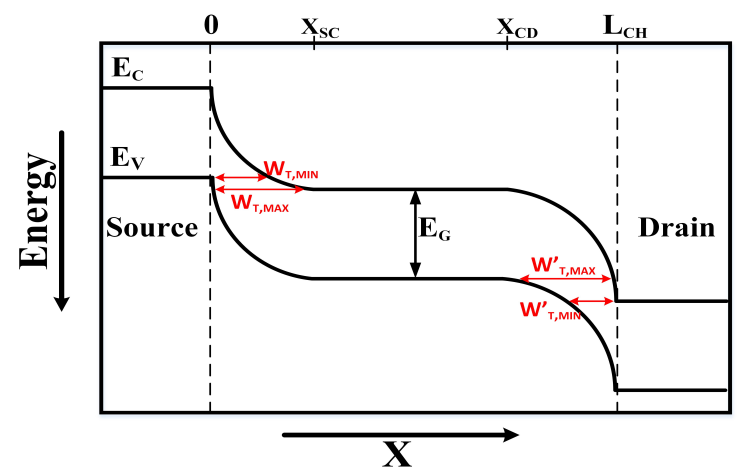

Figure 2: The energy bands in a tunneling field effect transistor indicating the maximum and minimum of the tunneling width in the source-channel and channel-drain junctions 12 . $X_{S C}$ and $X_{C D}$ represent the source and drain tunneling junction width.

$$
\frac{d^{2} \phi_{s}(x)}{d x^{2}}-\frac{\phi_{s}(x)-\left(V_{G S}-V_{f b}+P_{d} \frac{d_{f}}{\epsilon_{0} \epsilon_{f}}\right)}{\lambda^{2}}=\frac{q N_{c h}}{\epsilon_{s i}},
$$

where $\lambda=\sqrt{\epsilon_{s i} t_{s i} / C_{\text {stack }}}\left(t_{s i}\right.$ and $\epsilon_{s i}$ represent the silicon thickness and relative permittivity) is the characteristic length of the channel [12]. Solving (8) by considering the boundary conditions and the continuity of potential and electric field at the source-channel and channel-drain junctions, the surface potential profile around tunneling junctions can be obtained as

$$
\begin{gathered}
\phi_{s}(x)=\left(V_{G S(e f f)}\right)+\left(\phi_{c h}-V_{G S(e f f)}\right) \cosh \left(\frac{x-x_{s c}}{\lambda}\right) \cdots 0 \leq x \leq x_{s c} \\
\phi_{s}(x)=\left(V_{G S(e f f)}\right)+\left(\phi_{c h}-V_{G S(e f f)}\right) \cosh \left(\frac{x-x_{s d}}{\lambda}\right) \cdots x_{c d} \leq x \leq L_{c h} \\
x_{s c}=\lambda \cosh \left(\frac{V_{S 0}-\left(V_{G S(e f f)}\right)}{\phi_{c h}-\left(V_{G S(e f f)}\right)}\right) \\
x_{c d}=L_{c h}-\lambda \cdot \cosh ^{-1}\left(\frac{V_{D(e f f)}-\left(V_{G S(e f f)}\right)}{\phi_{c h}-\left(V_{G S(e f f)}\right)}\right)
\end{gathered}
$$


In the performed equations, $V_{S 0}$ subscribes to the source potential, $V_{D(e f f)}$ is the potential in the drain region $\left(V_{D(e f f)}=V_{S 0}+V_{b i, S D}+V_{D S}\right), V_{G S(e f f)}$ is the gate effective voltage $\left(V_{G S(e f f)}=V_{G S}-V_{f b}+P_{d}\left(d_{f} / \epsilon_{0} \epsilon_{f}\right)-\left(q N_{c h} / \epsilon_{s i}\right) \lambda^{2}\right)$, and $\phi_{c h}$ is the surface potential in the center of the channel that we have derived before. The tunneling width at the source junction can be expressed as

$$
\begin{aligned}
W_{t, \min }= & x\left(V_{S 0}+\frac{E_{g}}{q}\right)-x\left(V_{S 0}\right)= \\
& x_{s c}-\lambda \cdot \cosh ^{-1}\left(\frac{V_{D(e f f)}-\left(V_{G S(e f f)}\right)}{\phi_{c h}-\left(V_{G S(e f f)}\right)}\right), \\
W_{t, \max }= & x\left(\phi_{c h}\right)-x\left(\phi_{c h}-\frac{E_{g}}{q}\right)= \\
& \lambda \cosh ^{-1}\left(\frac{V_{S 0}+\frac{E_{g}}{q}-\left(V_{G S(e f f)}\right)}{\phi_{c h}-\left(V_{G S(e f f)}\right)}\right) .
\end{aligned}
$$

where $W_{t, \min }$ and $W_{t, \max }$ are the minimum and maximum of the tunneling width along the lateral tunneling path at the source-channel junction (the source-channel and channel-drain tunneling widthes are schematically depicted in Fig. 2). Regarding Kane's model [26, the tunneling current can be calculated by integrating the tunneling probability over the effective tunneling length,

$$
I_{\text {tunnel }, S \rightarrow C}=q . W . \int_{W_{t, \min }}^{W_{t, \max }} \int_{0}^{t_{s i}} G_{\text {Kane }} d y d x .
$$

In (15), $G_{K a n e}$ is the Kane's tunneling probability factor. The tunneling current at the drain junction can be calculated similarly,

$$
\begin{gathered}
W_{t, \text { min }}^{\prime}=x\left(V_{D(e f f)}\right)-x\left(V_{D(e f f)}-\frac{E_{g}}{q}\right), \\
W_{t, \max }^{\prime}=x\left(\phi_{c h}+\frac{E_{g}}{q}\right)-x\left(\phi_{c h}\right) .
\end{gathered}
$$




$$
I_{\text {tunnel }, C \rightarrow D}=q . W . \int_{W_{t, \min }^{\prime}}^{W_{t, \max }^{\prime}} \int_{0}^{t_{s i}} G_{\text {Kane }} d y d x
$$

135

the channel-drain junction. Finally, the total value of the device output current can be expressed as,

$$
I_{D S}=I_{\text {tunnel }, S \rightarrow C}+I_{\text {tunnel }, C \rightarrow D}
$$

\subsection{Dipole Polarization}

The value of the dipole polarization in each step is a function of the history of the applied electric field. Therefore, the polarization is determined by integrating $d P_{d}\left(E_{f}\right) / d E_{f}$ from a specified initial condition. Since this form has been extensively discussed before for the arbitrary field histories [17], the results are simply stated here. The saturated polarization hysteresis loop is defined by

$$
P_{\text {sat }}^{+}\left(E_{f}\right)=P_{s} \tanh \left(\frac{E_{f}-E_{C}}{2 \delta}\right), P_{\text {sat }}^{-}=-P_{\text {sat }}^{+}(-E)
$$

where

$$
\delta=E_{c}\left[\ln \left(\frac{1+\frac{P_{r}}{P_{s}}}{1-\frac{P_{r}}{P_{s}}}\right)\right],-1
$$

and $+($ or - ) superscript signifies the positive (or negative) going branch of the loop. The derivative of the polarization is given by the following equations:

$$
\begin{gathered}
\frac{d P_{d}}{d E_{f}}=\Gamma \frac{d P_{s a t}}{d E_{f}}, \\
\Gamma=1-\tanh \left[\left(\frac{P_{d}-P_{s a t}}{\xi P_{s}-P_{d}}\right)^{\frac{1}{2}}\right] .
\end{gathered}
$$

where $\xi=+1$ when $d E / d t>0$ and $\xi=-1$ when $d E / d t<0$.

Considering (1) to (19), the Fe-TFET drain current can be calculated as a function of the gate voltage history, device geometry, and material properties 
while the dipole polarization can be obtained using (20) to (23). In the following section, we will present a numerical analysis technique in order to obtain the Fe-TFET transfer characteristic.

\subsection{Numerical Analysis Technique}

Since the ferroelectric polarization depends on the history of the electric field, the polarization can be obtained by integrating from a known value. For the first step, we should define the initial values for the gate voltage and the polarization. Note that the initial conditions must satisfy the physical requirement that the polarization lies on or within the hysteresis loop. We considered $V_{g}=V_{f b}$ and $P_{d}=0$ as the initial condition. Having the polarization and the gate voltage, all TFET parameters are calculated using (1) to (19). Furthermore, the ferroelectric field and the silicon surface charge density can be calculated as follows:

$$
\begin{gathered}
\sigma_{s}=-\sqrt{2 \epsilon_{s i} k T N_{c h}}\left\{q \frac{\phi_{c h}}{k T}+\left(\frac{n_{i}}{N_{c h}}\right)^{2} \exp \left(q \frac{\phi_{c h}-V}{k T}\right)\right\}^{\frac{1}{2}}, \\
E_{f}=-\frac{\sigma_{s}+P_{d}(f)}{\epsilon_{0} \epsilon_{f}} .
\end{gathered}
$$

The approach to perform the integration from the initial conditions to a new set of conditions is to compute the $m$ 'th value of the relevant quantities, and numerically integrate. We now introduce subscript $m$, which signifies that the given quantity is evaluated at the $m$ 'th integration increment. First, we should solve equations (1) to (19) to find the $m$ 'th value of the surface potential. Then, we calculate the $m$ 'th value of the silicon surface charge and the electric field inside the ferroelectric film using equations (24) and (25). The polarization can be expressed as

$$
P_{d}\left(E_{m+1}\right)=P_{d}\left(E_{m}\right)+\left(E_{m+1}-E_{m}\right) \frac{d}{d E}\left[P_{d}(E)\right]_{E_{m}} .
$$

Considering (22) and (25), equation (26) can be rewritten as 

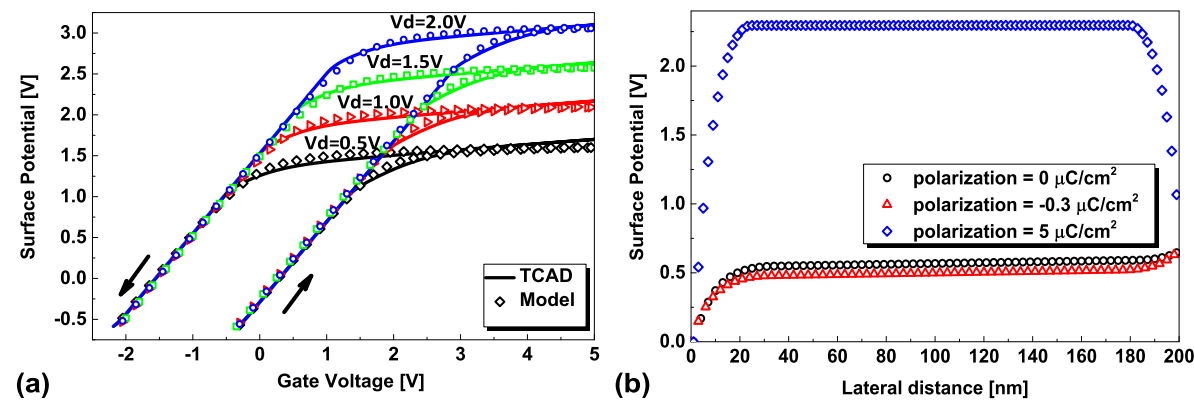

Figure 3: Ferroelectric TFET surface potential. (a) The model verification of the surface potential in the middle of the channel using different drain voltages and sweeping the gate voltage with Sentaurus TCAD simulations results. (b) The surface potential profile along the channel with the polarization as a parameter. Results are obtained using the proposed model for a device with $V_{G}=1 \mathrm{~V}, V_{D}=1.5 \mathrm{~V}$, and considering the gate oxide has $0,-0.003$, and $0.05 \mathrm{C} / \mathrm{m}^{2}$ polarization to illustrate how the positive and negative values of the polarization affect the potential profile.

$$
P_{d}\left(E_{m+1}\right)=\frac{P_{d}\left(E_{m}\right)-\left\{\left[\frac{\sigma_{s}}{\epsilon_{0} \epsilon_{f}}\right]+E_{m}\right\}\left[\left(\frac{d}{d E}\right) P_{d}(E)\right]_{E_{m}}}{1+\left(\frac{1}{\epsilon_{0} \epsilon_{f}}\right)\left[\left(\frac{d}{d E}\right) P_{d}(E)\right]_{E_{m}}} .
$$

The next value of the polarization in each step can be obtained using (27). Sweeping the gate voltage and calculating the next value of the polarization in each step, the drain current is calculated as a function of the gate voltage.

The model predicted results were verified by comparing with TCAD simulation resuls. The device surface potential is presented in Fig. (3). First, we verified the surface potential in the middle of the channel which is the primary parameter to compute the potential profile and the drain current. The surface potential profile in the middle of the channel for different drain voltages and sweeping the gate voltage is illustrated in Fig. 3-(a). Results confirm that our proposed model replicates well with the TCAD simulations. The effect of the dipole polarization on the surface potential profile along the channel is presented in Fig. 3(b). The surface potential profile is calculated for a TFET with $1 V$ gate voltage and $1.5 \mathrm{~V}$ drain voltage considering the gate oxide performs no ferroelectricity, ferroelectric with $-0.003 \mathrm{C} / \mathrm{m}^{2}$, and $0.05 \mathrm{C} / \mathrm{m}^{2}$ values of polarization to 

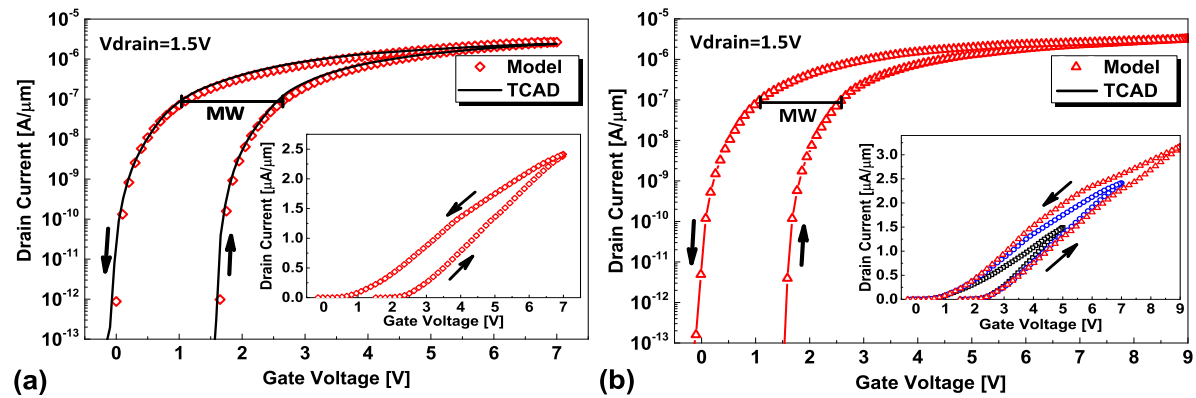

Figure 4: The ferroelectric TFET transfer characteristic for differnet drain and gate voltages. (a) The Fe-TFET model vs. TCAD results obtained for $1.5 \mathrm{~V}$ drain voltage. (b) Fe-TFET drain current for different sweeps of the gate voltage.

illuminate the effect of the positive and negative values of the polarization on the potential profile. Finally, the output current of the device is shown in Fig. (4). Fig. 4-(a) represents the output current while the drain voltage is $1.5 \mathrm{~V}$. The device's transfer characteristic for $1.5 \mathrm{~V}$ drain voltage and different sweeps of the gate voltage is illustrated in Fig. 4-(b).

\section{Memory Window}

The memory window (MW), a critical parameter in nonvolatile memory devices, is the threshold voltage difference between the two states of the device. Here, the constant current method at $10^{-7} \mathrm{~A} / \mathrm{um}$ is utilized for threshold voltage extraction [27. The memory window should be large enough to ensure a significant retention time and ease of data detection in nonvolatile memories. In this section, we discuss the quantitative influence of different device parameters that affect the memory window based on our presented model and theoretically highlight the advantages of the silicon-doped $\mathrm{HfO}_{2}$ ferroelectric thin film.

\subsection{Ferroelectric material}

It is well known that a Fe-FET memory window while the ferroelectric layer contains a sufficient remanent polarization and having the electric field higher than the material's coercive field mostly depends on the ferroelectric coercive 

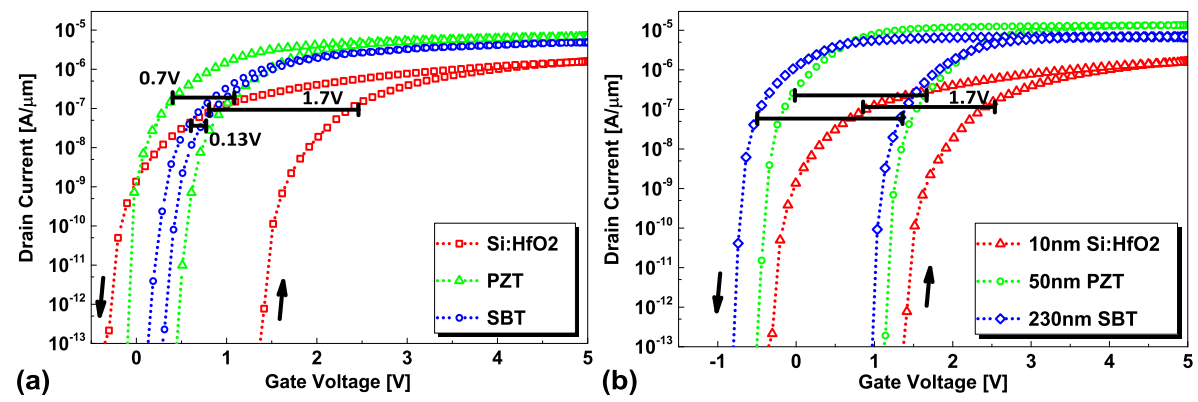

Figure 5: (a) The transfer characteristic of Fe-TFETs using $10 \mathrm{~nm}$ of PZT, SBT, and Si:HfO $\mathrm{H}_{2}$ as the gate ferroelectric obtained by our proposed model. The device benefits from doped $\mathrm{HfO}_{2}$ thin film provides larger memory window due to its high coercive field while all ferroelectrics has the same thickness. (b) The transfer characteristic of FeTFETs using different thicknesses of mentioned ferroelectric materials providing the same memory window. $10 \mathrm{~nm}$ of $\mathrm{Si}: \mathrm{HfO}_{2}$, $50 \mathrm{~nm}$ of PZT, and $230 \mathrm{~nm}$ of SBT are required to provide $1.7 \mathrm{~V}$ memory window.

field and thickness. In this subsection, we investigate the effect of the ferroelectric coercive field on the Fe-TFET memory window using the same thickness of different ferroelectrics. The properties of two well-known perovskite ferroelectrics, Lead Zirconate Titanate (PZT) and Strontium Barium Titanate (SBT), is compared with $\mathrm{Si}: \mathrm{HfO}_{2}$ in Table 1. The recently discovered ferroelectric thin film, Si: $\mathrm{HfO}_{2}$ 28, 6, has a relatively high coercive field that ensures a

large memory window [29, 30, 31, 32, [33, 34, 35, 36, 37, 38, 39].

Table 1: The ferroelectric properties of PZT [29, 30, 31, 32], SBT [33, 34, 35, 36, 37, 38, 39], and $\mathrm{Si}: \mathrm{HfO}_{2}$ [28].

\begin{tabular}{|c|c|c|c|c|}
\hline Ferro Material & $P_{r}\left(\frac{\mu C}{c^{2}}\right)$ & $P_{s}\left(\frac{\mu C}{c m^{2}}\right)$ & $E_{c}\left(\frac{M V}{c m}\right)$ & $\epsilon_{r}$ \\
\hline PZT & 32 & 40 & 0.26 & 250 \\
\hline SBT & 8 & 15 & 0.08 & 250 \\
\hline $\mathrm{Si}: \mathrm{HfO}_{2}$ & 9 & 9.5 & 1.1 & 32 \\
\hline
\end{tabular}

The $\mathrm{I}_{d}-V_{g}$ characteristics of a Fe-TFET using $10 \mathrm{~nm}$ of PZT, SBT, and $\mathrm{Si}: \mathrm{HfO}_{2}$ are depicted in Fig. 5-(a). We have used the same thickness of each material to eliminate the influence of the ferroelectric thickness on the MW. The Fe-TFET that is using PZT or SBT as the gate ferroelectric provides higher 
high dielectric constant of PZT and SBT reduces the voltage drop across the ferroelectric layer and enhances the silicon surface potential. Therefore, considering (11)-(14), the tunneling width at the source and drain tunneling junctions increases which lead to the improved tunneling current.

220 larger memory window compared to the well-known ferroelectric materials PZT and SBT. Up to $1.7 \mathrm{~V}$ memory window is obtained for a device using $10 \mathrm{~nm}$ of Si:HfO $\mathrm{H}_{2}$. In Fig. 5-(b) different thicknesses of PZT and SBT are used to maintain the same memory window as the Fe-TFET using $10 \mathrm{~nm}$ of $\mathrm{Si}: \mathrm{HfO}_{2}$. while $10 \mathrm{~nm}$ of silicon-doped $\mathrm{HfO}_{2}$ grant the same memory window. The theoretical results provide a great insight into the benefits of the silicon-doped $\mathrm{HfO}_{2}$ ferroelectric thin film. Besides the theoretical advantages of $\mathrm{Si}: \mathrm{HfO}_{2}$ comparing perovskite ferroelectrics, due to the high relative permittivity of the PZT perovskites ferroelectrics. Moreover, CMOS compatibility, scalability and the ability to deposit a high-quality layer by Atomic Layer Deposition (ALD) are some of the technological advantages of using $\mathrm{Si}: \mathrm{HfO}_{2}$.

The presented MWs in Fig. 5 are lower than the theoretical maximum of the ferroelectric field effect devices memory window $\left(\sim 2 E_{c} \times d\right.$ which is $2.2 \mathrm{~V}$ in the case of $10 \mathrm{~nm}$ Si:HfO 2 ) due to the depolarization field and gate leakage current effects. The depolarization field always exists in a ferroelectric capacitor due to the finite dielectric constant of the semiconductor that causes incomplete charge compensation. Moreover, the injected electrons from the gate and semiconductor to the ferroelectric layer lead to the local charge compensation and reducing polarization [40].

\subsection{Buffer layer}

Due to the large number of defects, leakage current, and large lattice mismatch between perovskite ferroelectrics and silicon, it is always necessary to use 


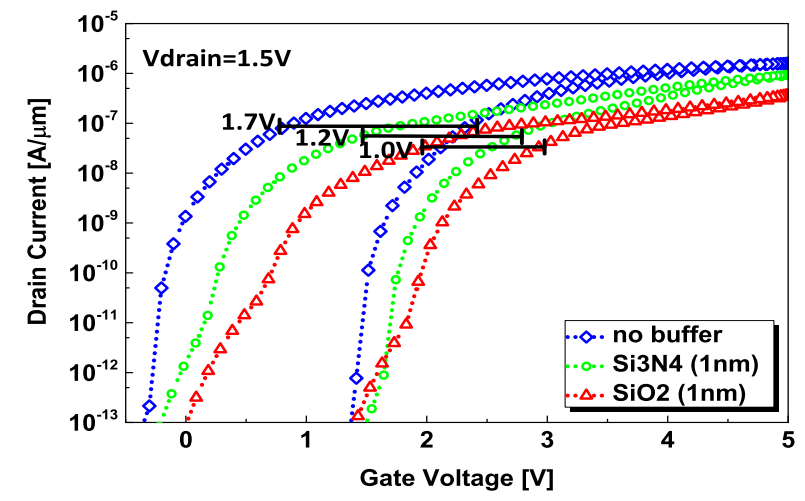

Figure 6: The output characteristic of a Fe-TFET using $10 \mathrm{~nm}$ of $\mathrm{Si}: \mathrm{HfO}_{2}$ as the gate stack with devices using $10 \mathrm{~nm}$ of $\mathrm{Si}: \mathrm{HfO}_{2}$ and $1 \mathrm{~nm}$ of $\mathrm{SiO}_{2}$ and $\mathrm{Si}_{3} \mathrm{~N}_{4}$ as the buffer layer. The device without buffer layer provides $1.7 \mathrm{~V}$ memory window, $1 \mathrm{~nm}$ of $\mathrm{Si}_{3} \mathrm{~N}_{4}$ reduces the MW down to $1.2 \mathrm{~V}$, and $1 \mathrm{~nm}$ of $\mathrm{SiO}_{2}$ provides $1.0 \mathrm{~V}$ memory window. The presented results are derived by employing the proposed model.

a buffer layer with a proper interface with the substrate. However, the voltage drop over the buffer layer reduces the memory window.

To study the effect of the buffer layer on the MW, we utilized $10 \mathrm{~nm}$ of $\mathrm{Si}: \mathrm{HfO}_{2}$ as the ferroelectric dielectric and $1 \mathrm{~nm}$ of $\mathrm{SiO}_{2}$ and $\mathrm{Si}_{3} \mathrm{~N}_{4}$ as the buffer layer. Results are compared with a device using only $10 \mathrm{~nm}$ of $\mathrm{Si}: \mathrm{HfO}_{2}$ as the gate stack in Fig. 6. Employing $1 \mathrm{~nm}$ of $\mathrm{SiO}_{2}$ drops the $\mathrm{MW}$ to $1.0 \mathrm{~V}$ while the use of $1 \mathrm{~nm}$ of $\mathrm{Si}_{3} \mathrm{~N}_{4}$ reduces the memory window to $1.2 \mathrm{~V}$ as the voltage drop over the buffer layer reduces the ferroelectric voltage (electric field). The voltage drop over the buffer layer is considerable as $\mathrm{SiO}_{2}$ and $\mathrm{Si}_{3} \mathrm{~N}_{4}$ have lower relative permittivity comparing to the ferroelectric layer. Reducing ferroelectric voltage results in the formation of minor polarization hysteresis loops instead of the saturation polarization loop that reduces the memory window significantly [16, 13. Moreover, the presence of the buffer layer reduces the silicon surface potential results in low energy carriers and insufficient tunneling through the source and drain tunneling barriers that lead to the deformation of the transfer characteristic and reduced saturation current. The negative influence of the buffer layer can be reduced in the case of high-K materials that lowers the 
voltage drop across this layer, but it can never be zero. In this subsection, we emphasize on another advantage of $\mathrm{Si}: \mathrm{HfO}_{2}$ which is its proper interface with silicon. Therefore, the doped $\mathrm{HfO}_{2}$ thin film can be deposited directly on the silicon, and no buffer layer is required. However, it should be noted that a thin layer of HfSiO forms at the interface during the deposition and annealing of the $\mathrm{Si}: \mathrm{HfO}_{2}$ which is called the dead layer. The dead layer performs no ferroelectricity, and it behaves like a buffer layer causing reduced memory window 6, 28. As the dead layer is a related issue for all ferroelectric materials we have neglected this effect in our simulations.

Besides reducing the memory window, presence of the buffer layer also increases the subthreshold slope affecting the response time and power dissipation.

\subsection{Ferroelectric thickness}

Another important parameter that greatly affects the memory window is the ferroelectric thickness. The ferroelectric thickness strongly affects the MW as it specifies the total number of the dipoles $\left(M W \sim 2 E_{c} \times d\right.$ where $E_{c}$ and $d$ are the ferroelectric coercive field and thickness relatively [40]). Furthermore, the ferroelectric coercive field might change by varying the film thickness, especially in the case of the doped $\mathrm{HfO}_{2}$. Increasing the films thickness in $\mathrm{Si}: \mathrm{HfO}_{2}$ reduces the coercive field. The ferroelectric properties of the $\mathrm{Si}: \mathrm{HfO}_{2}$ regarding the film thickness is depicted in Table 2.

The transfer characteristic of the Fe-TFET using different thicknesses of $\mathrm{Si}: \mathrm{HfO}_{2}$ is illustrated in Fig. 7. The coercive field slightly reduces by increasing the silicon-doped $\mathrm{HfO}_{2}$ thickness. However, simulation results confirm that the

Table 2: The ferroelectric properties of $\mathrm{Si}: \mathrm{HfO}_{2}$ regarding the fims thickness [6, 28].

\begin{tabular}{c|cccc} 
Thickness & $P_{r}\left(\frac{\mu C}{c m^{2}}\right)$ & $P_{s}\left(\frac{\mu C}{c m^{2}}\right)$ & $E_{c}\left(\frac{M V}{c m}\right)$ & $\epsilon_{r}$ \\
\hline \hline 20nm & 5.5 & 5.75 & 1.00 & 28.5 \\
$\mathbf{1 5 n m}$ & 7.25 & 7.62 & 1.05 & 30.25 \\
$\mathbf{1 0 n m}$ & 9 & 9.5 & 1.1 & 32
\end{tabular}




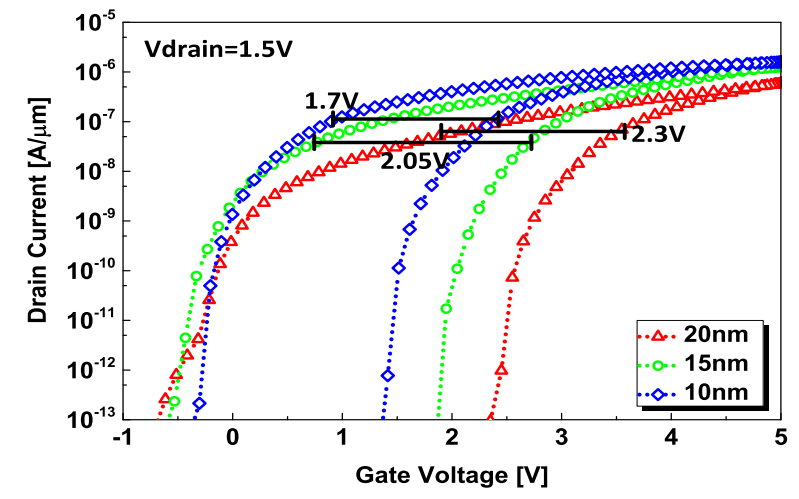

Figure 7: The $I_{d}-V_{g}$ curves of the Fe-TFETs using different thicknesses of Si:HfO ${ }_{2}$. Results are obtained using our proposed model.

effect of the film thickness is dominant and the memory window enhances significantly by increasing the ferroelectric thickness. Up to $2.7 \mathrm{~V}$ memory window is obtained for a device benefits from a $20 \mathrm{~nm}$ layer thick $\mathrm{Si}: \mathrm{HfO}_{2}$.

\section{Nanometer scale Fe-TFET}

Unique properties of the ferroelectric $\mathrm{Si}: \mathrm{HfO}_{2}$ like relatively low dielectric constant, CMOS compatibility, good interface with the silicon [7, and relatively high remanent polarization even in a $5 \mathrm{~nm}$ thick, thin film [15] make it a promising candidate for the fabrication of future nonvolatile memories [28, 6].

The unique properties of $\mathrm{Si}: \mathrm{HfO}_{2}$ allows us to fabricate ferroelectric MOSFETs down to the nanoscale. However, on the nanometer scale, the high power consumption per area of the chip becomes a challenging issue. To eliminate the power dissipation problem we need to design energy efficient devices with low off-current. Tunneling field effect transistors have been proposed as energy efficient devices due to their low off-current and steep off to on transition. The Fe-TFET can be a decent candidate for future nonvolatile memories due to its ability to provide a sufficient memory window with low power dissipation. As we did not consider short channel effects in our model, results of the model may not be reliable for a nanometer scale device. Therefore, Sentaurus TCAD com- 

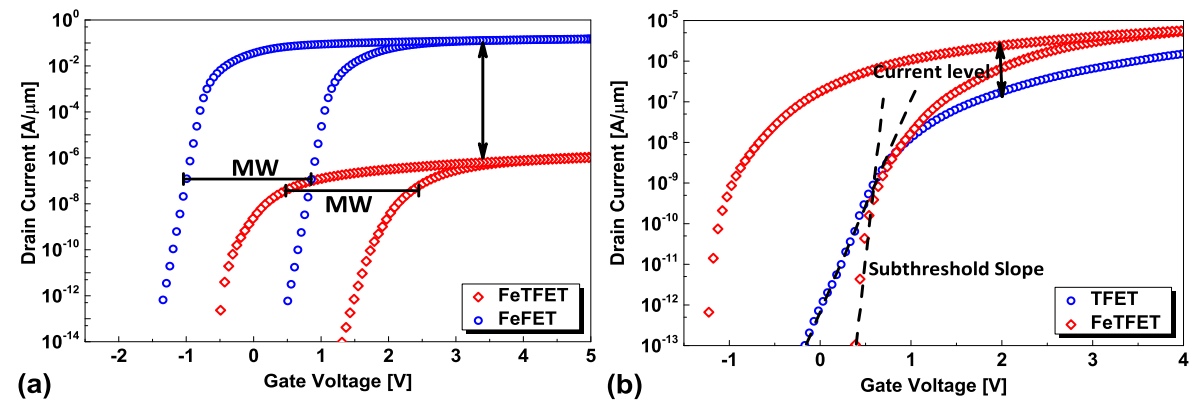

Figure 8: (a) The transfer characteristic of a $28 \mathrm{~nm}$ gate length FeFET vs. Fe-TFET using $10 \mathrm{~nm}$ of $\mathrm{Si}: \mathrm{HfO}_{2}$ as the ferroelectric. The drain voltage is considered $1 \mathrm{~V}$ for all simulations which is the supply voltage of the $28 \mathrm{~nm}$ ITRS node. Results confirm that the Fe-TFET provides the same memory window as the FeFET with lower power consumption. (b) The negative capacitance effect enhances the output current level and subthreshold slope of the Fe-TFET comparing the conventional TFET. Results are obtained using Sentaurus TCAD commercial simulator.

mercial simulator is employed for design and simulation of a $28 \mathrm{~nm}$ gate length Fe-TFET.

In Fig. 8-(a), the transfer characteristic of a $28 \mathrm{~nm}$ gate length single gate ferroelectric MOSFET is compared with Fe-TFET with the same dimensions. $10 \mathrm{~nm}$ of $\mathrm{Si}: \mathrm{HfO}_{2}$ is used as the gate ferroelectric. Regarding the presented results in Fig. 8-(a), Fe-TFET provides the same memory window as FeFET with lower off-current (static power) and also lower on-current 40. However, the TFET low output current is the main disadvantage of this device as it may not be sufficient to drive the circuit capacitors. It should be noted that besides the ability of ferroelectrics for data storage, ferroelectric transistors also benefits from the ferroelectrics negative capacitance effect that amplifies the surface potential and boosts the output current [41, 42]. In Fig. 8-(b), the $I_{d}-V_{g}$ characteristic of a $28 \mathrm{~nm}$ Fe-TFET with $10 \mathrm{~nm}$ of $\mathrm{Si}: \mathrm{HfO}_{2}$ is compared with a $28 \mathrm{~nm}$ gate length conventional TFET with the same equivalent oxide thickness. As a result of the negative capacitance effect, the subthreshold slope of the device is decreased, and the drain current is increased significantly. Simulation results illustrate that the Fe-TFET meets our expectation for a low power random 
access nonvolatile memory providing sufficient memory window.

\section{Conclusions}

The analytical modeling of ferroelectric TFETs is developed and examined using Sentaurus TCAD commercial simulator. The model is obtained by solving Maxwell's first equation in conjunction with Poisson equation. Based on the proposed model, the memory window of the Fe-TFET memory devices is extensively investigated as the key parameter of nonvolatile memories. The recently discovered ferroelectric, silicon-doped $\mathrm{HfO}_{2}$, is integrated into the TFET gate stack in order to highlight the advantages of this ferroelectric thin film. Theoretical results represent that $\mathrm{Si}: \mathrm{HfO}_{2}$ stands as a promising candidate for the future of ferroelectric nonvolatile memories. Besides the outstanding properties of the $\mathrm{Si}: \mathrm{HfO}_{2}$ for the device fabrication, a large memory window is obtained as the result of the integration of this ferroelectric thin film with tunneling field effect transistors. Finally, a $28 \mathrm{~nm}$ gate length Fe-TFET is designed and simulated providing the same MW as a $28 \mathrm{~nm}$ Fe-FET consuming less power dissipation. 335 Results confirm that the Fe-TFET using $\mathrm{Si}: \mathrm{HfO}_{2}$ can be ustilized as the ultra-

low power nonvolatile memories. Moreover, the negative capacitnace effect in ferroelectric TFETs can solve the the tunneling devices main issue, low output current, by boosting the surface potential.

\section{References}

[1] A. M. Ionescu, L. Lattanzio, G. A. Salvatore, L. De Michielis, K. Boucart, D. Bouvet, The hysteretic ferroelectric tunnel fet, Electron Devices, IEEE Transactions on 57 (12) (2010) 3518-3524.

[2] S. Sakaia, M. Takahashia, K. Takeuchib, Q.-H. Lia, T. Horiuchia, S. Wanga, K.-Y. Yuna, M. Takamiyac, T. Sakuraic, Highly scalable fe (ferroelectric)nand cell with mfis (metal-ferroelectric-insulator-semiconductor) structure 
for sub-10nm tera-bit capacity nand flash memories, in: 2008 Joint NonVolatile Semiconductor Memory Workshop and International Conference on Memory Technology and Design, 2008.

[3] J. Müller, P. Polakowski, S. Müller, T. Mikolajick, Ferroelectric hafnium oxide based materials and devices: Assessment of current status and future prospects, ECS Journal of Solid State Science and Technology 4 (5) (2015) N30-N35.

[4] P. Heyman, G. Heilmeier, A ferroelectric field effect device, Proceedings of the IEEE 54 (6) (1966) 842-848.

[5] E. Tokumitsu, R.-i. Nakamura, H. Ishiwara, Nonvolatile memory operations of metal-ferroelectric-insulator-semiconductor (mfis) fets using plzt/sto/si (100) structures, Electron Device Letters, IEEE 18 (4) (1997) 160-162.

[6] T. Böscke, J. Müller, D. Bräuhaus, U. Schröder, U. Böttger, Ferroelectricity in hafnium oxide: Cmos compatible ferroelectric field effect transistors, in: Electron Devices Meeting (IEDM), 2011 IEEE International, IEEE, 2011, pp. $24-5$.

[7] S. Mueller, J. Muller, U. Schroeder, T. Mikolajick, Reliability characteristics of ferroelectric thin films for memory applications, Device and Materials Reliability, IEEE Transactions on 13 (1) (2013) 93-97.

[8] Q. Huang, R. Huang, Z. Zhan, Y. Qiu, W. Jiang, C. Wu, Y. Wang, A novel si tunnel fet with $36 \mathrm{mv} / \mathrm{dec}$ subthreshold slope based on junction depleted-modulation through striped gate configuration, in: Electron Devices Meeting (IEDM), 2012 IEEE International, IEEE, 2012, pp. 8-5.

[9] C. Hu, P. Patel, A. Bowonder, K. Jeon, S. H. Kim, W. Y. Loh, C. Y. Kang, J. Oh, P. Majhi, A. Javey, et al., Prospect of tunneling green transistor for 0.1 v cmos, IEDM Tech. Dig (2010) 16-1.

[10] A. M. Ionescu, H. Riel, Tunnel field-effect transistors as energy-efficient electronic switches, Nature 479 (7373) (2011) 329-337. 
[11] http://www.synopsys.com/tools/tcad/.

${ }_{375}$ [12] C. Wu, R. Huang, Q. Huang, C. Wang, J. Wang, Y. Wang, An analytical surface potential model accounting for the dual-modulation effects in tunnel fets, Electron Devices, IEEE Transactions on 61 (8) (2014) 2690-2696.

[13] S. Miller, R. Nasby, J. Schwank, M. Rodgers, P. Dressendorfer, Device modeling of ferroelectric capacitors, Journal of applied physics 68 (12) (1990) 6463-6471.

[14] D. Zhou, J. Müller, J. Xu, S. Knebel, D. Bräuhaus, U. Schröder, Insights into electrical characteristics of silicon doped hafnium oxide ferroelectric thin films, Applied Physics Letters 100 (8) (2012) 082905.

[15] S. Mueller, S. R. Summerfelt, J. Müller, U. Schroeder, T. Mikolajick, Tennanometer ferroelectric films for next-generation fram capacitors, Electron Device Letters, IEEE 33 (9) (2012) 1300-1302.

[16] H.-T. Lue, C.-J. Wu, T.-Y. Tseng, Device modeling of ferroelectric memory field-effect transistor (femfet), Electron Devices, IEEE Transactions on 49 (10) (2002) 1790-1798.

[17] S. Miller, P. McWhorter, Physics of the ferroelectric nonvolatile memory field effect transistor, Journal of applied physics 72 (12) (1992) 5999-6010.

[18] S. Miller, J. Schwank, R. Nasby, M. Rodgers, Modeling ferroelectric capacitor switching with asymmetric nonperiodic input signals and arbitrary initial conditions, Journal of applied physics 70 (5) (1991) 2849-2860.

[19] M. J. Kumar, S. Janardhanan, Doping-less tunnel field effect transistor: design and investigation, Electron Devices, IEEE Transactions on 60 (10) (2013) 3285-3290.

[20] S. James, P. Arujo, A. Carlos, Ferroelectric memories, Science 246 (4936) (1989) 1400-1405. 
[21] R. Zuleeg, H. Wieder, Effect of ferroelectric polarization on insulated-gate thin-film transistor parameters, Solid-State Electronics 9 (6) (1966) 657661.

[22] L. Landau, I. Khalatnikov, On the anomalous absorption of sound near a second order phase transition point, in: Dokl. Akad. Nauk SSSR, Vol. 96, 1954, pp. 469-472.

[23] V. C. Lo, Simulation of thickness effect in thin ferroelectric films using landau-khalatnikov theory, Journal of applied physics 94 (5) (2003) 33533359 .

[24] W. Zhang, K. Bhattacharya, A computational model of ferroelectric domains. part i: model formulation and domain switching, Acta materialia 53 (1) (2005) 185-198.

[25] R.-H. Yan, A. Ourmazd, K. F. Lee, Scaling the si mosfet: from bulk to soi to bulk, Electron Devices, IEEE Transactions on 39 (7) (1992) 1704-1710.

[26] E. Kane, Zener tunneling in semiconductors, Journal of Physics and Chemistry of Solids 12 (2) (1960) 181-188.

[27] K. Boucart, A. M. Ionescu, Threshold voltage in tunnel fets: physical definition, extraction, scaling and impact on ic design, in: Solid State Device Research Conference, 2007. ESSDERC 2007. 37th European, IEEE, 2007, pp. 299-302.

[28] S. Mueller, E. Yurchuk, S. Slesazeck, T. Mikolajick, J. Muller, T. Herrmann, A. Zaka, Performance investigation and optimization of si: Hfo 2 fefets on a $28 \mathrm{~nm}$ bulk technology, in: Applications of Ferroelectric and Workshop on the Piezoresponse Force Microscopy (ISAF/PFM), 2013 IEEE International Symposium on the, IEEE, 2013, pp. 248-251.

${ }_{425}$ [29] I. Kanno, S. Fujii, T. Kamada, R. Takayama, Piezoelectric properties of c-axis oriented pb (zr, ti) o3 thin films, Applied Physics Letters 70 (11) (1997) 1378-1380. 
[30] M. Grossmann, O. Lohse, D. Bolten, U. Boettger, T. Schneller, R. Waser, The interface screening model as origin of imprint in pbzrxti1- xo3 thin films. i. dopant, illumination, and bias dependence, Journal of applied physics 92 (5) (2002) 2680-2687.

[31] R. Moazzami, C. Hu, W. H. Shepherd, Electrical characteristics of ferroelectric pzt thin films for dram applications, Electron Devices, IEEE Transactions on 39 (9) (1992) 2044-2049.

[32] T. Nakamura, Y. Nakao, A. Kamisawa, H. Takasu, Preparation of pb (zr, ti) o3 thin films on electrodes including iro2, Applied physics letters 65 (12) (1994) 1522-1524.

[33] K. Amanuma, T. Hase, Y. Miyasaka, Preparation and ferroelectric properties of srbi2ta2o9 thin films, Applied physics letters 66 (2) (1995) 221-223.

${ }_{440}$ [34] H. Al-Shareef, D. Dimos, T. Boyle, W. Warren, B. Tuttle, Qualitative model for the fatigue-free behavior of srbi2ta2o9, Applied physics letters 68 (5) (1996) 690-692.

[35] Z. Zhang, Y. Wang, J. Zhu, F. Yan, X. Lu, H. Shen, J. Liu, Retention characteristics of srbi2ta2o9 thin films prepared by metalorganic decomposition, Applied physics letters 73 (25) (1998) 3674-3676.

[36] H. N. Lee, A. Visinoiu, S. Senz, C. Harnagea, A. Pignolet, D. Hesse, U. Gosele, Structural and electrical anisotropy of (001)-,(116)-, and (103)oriented epitaxial srbi2ta2o9 thin films on srtio3 substrates grown by pulsed laser deposition, Journal of Applied Physics 88 (11) (2000) 6658-6664.

450

[37] T. Mikolajick, C. Dehm, W. Hartner, I. Kasko, M. Kastner, N. Nagel, M. Moert, C. Mazure, Feram technology for high density applications, Microelectronics Reliability 41 (7) (2001) 947-950.

[38] M. Mört, G. Schindler, W. Hartner, I. Kasko, M. J. Kastner, T. Mikolajick, C. Dehm, R. Waser, Low temperature process and thin sbt films 
for ferroelectric memory devices, Integrated Ferroelectrics 30 (1-4) (2000) 235-244.

[39] W. Hartner, G. Schindler, P. Bosk, Z. Gabric, M. Kastner, G. Beitel, T. Mikolajick, C. Dehm, C. Mazuré, Integration of h2 barriers for ferroelectric memories based on srbi2ta2o9 (sbt), Integrated Ferroelectrics 31 (1-4) (2000) 273-284.

[40] T. Ma, J.-P. Han, Why is nonvolatile ferroelectric memory field-effect transistor still elusive?, Electron Device Letters, IEEE 23 (7) (2002) 386-388.

[41] A. Rusu, G. A. Salvatore, D. Jimenez, A. M. Ionescu, Metal-ferroelectricmeta-oxide-semiconductor field effect transistor with sub-60mv/decade subthreshold swing and internal voltage amplification, in: Electron Devices Meeting (IEDM), 2010 IEEE International, IEEE, 2010, pp. 16-3.

[42] S. Salahuddin, S. Datta, Use of negative capacitance to provide voltage amplification for low power nanoscale devices, Nano letters 8 (2) (2008) 405-410. 